\title{
Verbascum kurdistanicum (Scrophulariaceae), a new species from Hakkâri, Turkey
}

\author{
Mehmet Firat' \\ I Yüzüncü Yıl University, Faculty of Education, Department of Biology, 65080 Van, Turkey \\ Corresponding author: Mehmet Firat (kuyucak65@yahoo.com)
}

Academic editor: Alan Paton | Received 25 April 2015 | Accepted 22 June 2015 | Published 8 July 2015

Citation: Fırat M (2015) Verbascum kurdistanicum (Scrophulariaceae), a new species from Hakkâri, Turkey. PhytoKeys 52: 89-94. doi: 10.3897/phytokeys.52.5188

\begin{abstract}
Verbascum kurdistanicum Firat (Scrophulariaceae), is described and illustrated as a new species that is located in Hakkâri, Turkey. In this study, diagnostic morphological characters of this and closely related species ( $V$. oreophilum K.Koch and V. pyramidatum M. Bieb) are discussed. Furthermore, distribution maps for the three taxa are provided.
\end{abstract}

\section{Keywords}

New species, Hakkâri , Turkey, Verbascum

\section{Introduction}

Verbascum L. (Scrophulariaceae) includes about 360 species distributed throughout the world (Heywood 1993). This genus in Turkey includes about 245 species, 129 hybrids and 6 imperfectly known or doubtful records. Its endemism ratio is very high with 193 (79\%) species restricted to Turkey (Huber-Morath 1978, Davis et al. 1988, Vural and Aydoğdu 1993, Karavelioğulları et al. 2004, 2006, 2008a, 2009a, 2009b, 2011, 2014, Karavelioğulları 2012, Sutorý 2001, 2004, Özhatay 2006, Kaynak et al. 2006, Yilmaz and Dane 2008, Bani et al. 2010).

Verbascum is divided into two sections (Murbeck 1933, Huber-Morath 1971): Aulacospermae Murb. and Bothrospermae (Murb.) Kamelin. The seed morphology of their members is the most important character which differentiates the sections. They are transversely corrugated and alveolate in sect. Bothrospermae, whereas the seeds are

Copyright Mehmet Firat. This is an open access article distributed under the terms of the Creative Commons Attribution License (CC BY 4.0), which permits unrestricted use, distribution, and reproduction in any medium, provided the original author and source are credited. 
longitudinally corrugated in sect. Aulacospermae. Section Bothrospermae includes all Turkish Verbascum species.

The first revision of Turkish Verbascum for Flora of Turkey was carried out by HuberMorath (1978). Thirteen species and six hybrids were later described (Vural and Aydoğdu 1993, Karavelioğulları et al. 2004, 2006, 2008a, 2009a, 2011, 2014, Karavelioğulları 2012, Sutorý 2001, 2004, Özhatay 2006, Kaynak et al. 2006, Dane and Yilmaz 2009, Dane and Yılmaz 2005, Yilmaz and Dane 2008, Karavelioğulları 2009b).

\section{Materials and methods}

During field exploration in Hakkâri province, Turkey in 2011, an unusual population of Verbascum was discovered. At first glance, because of having corolla and capsule with branched eglandular hairs, glandular-hairy inflorescence and distinctly crenate lower leaves it seemed to be similar to $V$. oreophilum and $V$. pyramidatum. The specimens were cross-checked with the keys provided by Huber-Morath $(1978,1981)$ and the Verbascum accounts given in various relevant publications such as Fedchenko (1955), Feinbrun-Dothan (1978a, 1978b), Meikle (1985), Boulos (2009) and Ekim (2000). Herbarium specimens from VANF, GAZI, ANK, G and GB herbaria were also examined and compared. The threat category assessment of the new species was defined according to IUCN criteria (IUCN 2001).

\section{Taxonomy}

Verbascum kurdistanicum Firat, sp. nov. urn:Isid:ipni.org:names:77148112-1

Figs $1-2$

Type. TURKEY. C9 Hakkâri: Berçelan Plateau, 3740'57'N, 04343'21'E, 2600$2800 \mathrm{~m}$, limestone rocks and steppe, 21 July 2011, M. Firat. 27584. (Holotype: VANF, Isotype: ANK, GAZI, HUB, VANF, E).

Diagnosis. Verbascum kurdistanicum differs from $V$. oreophilum and $V$. pyramidatum by being biennial; having 8-30 (incl. petiole) $\times 2.5-4.5 \mathrm{~cm}$, lanceolate, crenate basal leaves; linear-lanceolate calyx lobes; 4 stamens; two anterior filaments that are glabrous near apex; 10-15 × 6-8 mm, ovate to oblong capsule.

Description. Biennial, 35-170 cm, densely stellate below, sparsely stellate, densely stalked glandular above. Stem robust, terete, erect, branched. Basal leaves 8-30 (inc. petiole) $\times 2.5-4.5 \mathrm{~cm}$, mostly congested at base, densely rosulate, lanceolate, entire, distinctly undulate, obtuse, gradually attenuate at base. Cauline leaves $2.5-4 \times 0.5-1$ $\mathrm{cm}$, oblong-lanceolate, entire, acute, decreasing in size towards the inflorescence racemose, ascending-erect. Bracts 2-3 × 1-2 mm, ovate-lanceolate, entire, acute, each bract with a solitary flower. Pedicels $5-10 \mathrm{~mm}$. Bracteoles absent. Calyx 3-10 mm, 


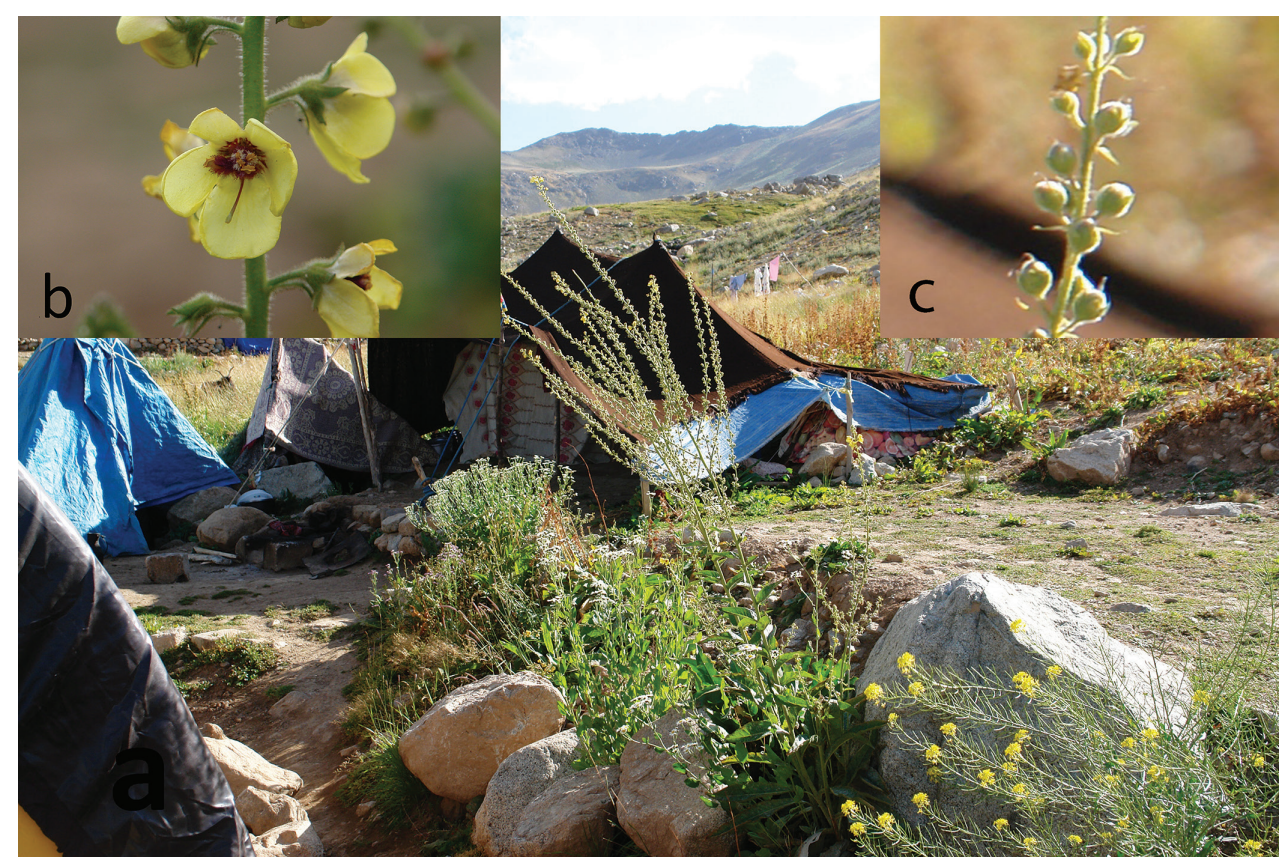

Figure I. Verbascum kurdistanicum Fırat: a habit b corolla c capsule.

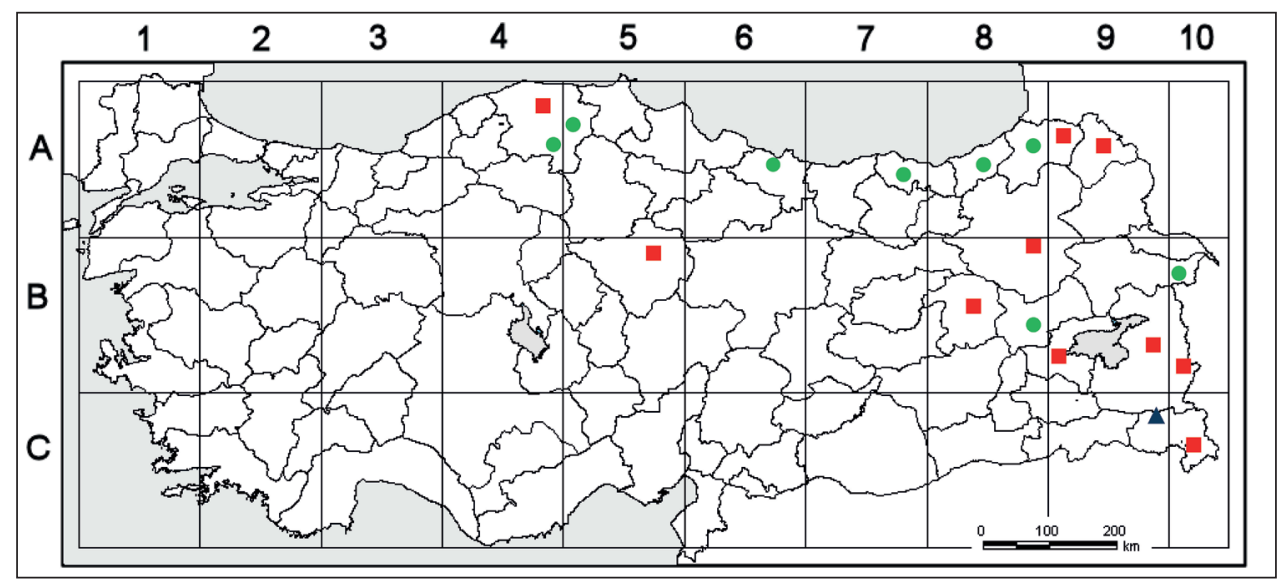

Figure 2. Distribution map of $V$. kurdistanicum $(\mathbf{\Delta})$ and also closely related species $V$. oreophilum ( $\mathbf{(})$ and $V$.pyramidatum $(\bullet)$.

divided almost to base, with linear-lanceolate acute lobes. Corolla 20-30 mm diam, yellow, tube 1-2 mm, with unequal and orbicular lobes, without pellucid-punctate glands, with sparsely stalked glandular, stellate outside. Stamens 4, 6-8 mm, filaments 5-6 mm, with purple-violet wool, two anterior glabrous near apex, anthers $1-2 \mathrm{~mm}$, reniform. Ovary ovate. Style 5-7 mm, filiform. Stigma spathulate. Capsule 10-15 × 6-8 mm, ovate, densely stellate hairs, rarely soon glabrescent. 
Flowering time June-July and Fruiting time July-August, limestone rocks and steppe, 2600-2800 m.

Vernacular name. In Hakkâri Province, indigenous people use the name "Masîjark" for Verbascum (Firat 2013).

\section{Examined representative specimens:}

Verbascum oreophilum: Turkey. C10 Hakkari: Van-Hakkâri 113. km, c. 2400 m, 19 July 1956, alpine meadow, H.Birand \& K.Karamanoğlu 471 (ANK).

Other herbarium materials of Verbascum oreophilum and Verbascum pyramidatum which were collected from Turkey have been examined. VANF, GAZI, ANK, G and GB herbarium have been visited for representative specimens.

Red list assessment: The extent of occurrence for Verbascum kurdistanicum was less than $100 \mathrm{~km}^{2}$ (approximately between 10-20 km²). 304 mature individuals have been counted. This species was found in a single location. It grows in limestone rocks and steppe. Its habitat continues to decline because of agricultural activities and other local uses. Hence, the threat category of Verbascum kurdistanicum is suggested as CR [B1ab (i, ii, iii) + 2ab (i, ii, iii)].

\section{Results and discussion}

Because of having 4 stamens, Verbascum kurdistanicum belongs to the group A according to the Flora of Turkey (Huber-Morath 1978, Davis et al. 1988). This group comprises 30 species with the addition of Verbascum kurdistanicum.

Verbascum kurdistanicum is morphologically similar to V. oreophilum and $V$. pyramidatum because of having the corolla and capsule with branched eglandular hairs; glandular-hairy inflorescence and distinctly crenate lower leaves, but differs from being biennial; having different basal leaves; stamen; flaments and capsule (Table 1).

Table I. Diagnostic characters of Verbascum kurdistanicum compared with the related V. oreophilum and V. pyramidatum.

\begin{tabular}{|c|c|c|c|}
\hline Characters & V. kurdistanicum & V. oreophilum & V. pyramidatum \\
\hline Plant & Biennial, 35-160 cm & Perennial, 60-160 cm & Perenial, $45-150 \mathrm{~cm}$ \\
\hline Basal leaves & $\begin{array}{c}8-30(\text { incl. petiole }) \times \\
2.5-4.5 \mathrm{~cm} \text {, lanceolate, } \\
\text { crenate }\end{array}$ & $\begin{array}{c}10-40 \times 5-20 \mathrm{~cm} \text {, lanceolate- } \\
\text { oblong to ovate-elliptic } \\
\text { coarsely crenate or bi crenate, } \\
\text { more rarely dentate-lobed }\end{array}$ & $\begin{array}{l}\text { 7-40 } \times 3-15 \mathrm{~cm} \text {, lanceolate- } \\
\text { oblong to obovate, coarsely } \\
\text { bicrenate, crenata-dentate or } \\
\text { weakly lobed }\end{array}$ \\
\hline Calyx & lobes linear-lanceolate & lobes oblong & lobes oblong \\
\hline Stamens & 4 & 4-rarely 5 & 5-rarely 4 \\
\hline Filaments & $\begin{array}{c}\text { two anterior glabrous } \\
\text { near apex }\end{array}$ & $\begin{array}{c}\text { two anterior woolly to anthers } \\
\text { or glabrous near apex }\end{array}$ & all woolly \\
\hline Capsule & $\begin{array}{c}10-15 \times 6-8 \mathrm{~mm} \text {, ovate } \\
\text { to oblong }\end{array}$ & $\begin{array}{c}4-6 \times 3-5.5 \mathrm{~mm} \text {, ellipsoid- } \\
\text { ovate or subglobose }\end{array}$ & $\begin{array}{c}4-8 \times 3-5 \mathrm{~mm} \text {, broadly } \\
\text { elliptic to ovate }\end{array}$ \\
\hline
\end{tabular}




\section{Acknowledgements}

Thanks to Dr. Faik Ahmet Karavelioğulları for support description and critical discussion about the manuscript.

\section{References}

Bani B, Adıguzel N, Karavelioğulları FA (2010) A new species (Verbascum turcicum sp. nov., Scrophulariaceae) from South Anatolia, Turkey. Annales Botanici Fennici 47: 489-492.

Boulos L (2009) Flora of Egypt Check list. Al-Hadara Publishing, Cairo. doi: $10.5735 / 085.047 .0608$

Brummitt RK, Powell CE (1992) Authors of Plant Names. Royal Botanic Gardens, Kew Publishing, $732 \mathrm{pp}$.

Dane F, Yılmaz G (2005) New records for the flora of European Turkey: Verbascum roripifolium and V. ovalifolium subsp. thracicum (Scrophulariaceae). Botanika Chronika 18(2): 11-16.

Dane F, Yilmaz G (2009) A new natural hybrid of Verbascum (Scrophulariaceae) from European Turkey. Phytologia Balcanica 15(2): 159-162.

Davis PH, Mill RR, Tan K (1988) Flora of Turkey and the East Aegean Islands (Suppl.1). Vol. 10. Edinburgh University Press, Edinburgh, 191-193.

Ekim T (2000) Verbascum L. In: Güner A, Ozhatay N, Ekim T, Başer KHC (Eds) Flora of Turkey and the East Aegean Islands (Suppl. 2). Vol. 11. Edinburgh University Press, Edinburgh, 193.

Fedchenko BA (1955) Verbascum L. In: Schischkin BK, Bobrow EG (Eds) Flora of U.S.S.R. Vol. 22. Izdatel'stvo Akademii Nauk S.S.S.R., Leningrad, 132-197.

Feinbrun-Dothan N (1978a) Verbascum L. In: Zohary M, Feinbrun-Dothan N (Eds) Flora Palaestina (Text). Vol. 3. Academic Press, Jerusalem, 282-302.

Feinbrun-Dothan N (1978b) Verbascum L. In: Zohary M, Feinbrun-Dothan N (Eds) Flora Palaestina (Plates).Vol. 3. Academic Press, Jerusalem, 170-182.

Fırat M (2013) Ferhenga Navên Riwekên Bi Kurdî/Kürtçe Bitki Adları Sözlüğü / Dictionary of Plant Names in Kurdish. Kalkan Ofset, Ankara, 1-652.

Heywood VH (1993) Flowering Plants of the World. Oxford University Press, New York.

Holmgren PK, Holmgren NH (1990) Index Herbariorum. New York Botanical Garden.

Huber-Morath A (1971) Die Türkishchen Verbasceen. Kommissionsverlag von Gebruder Fretz A. G. Zurih.

Huber-Morath A (1978) Verbascum L. In: Davis PH (Ed.) Flora of Turkey and the East Aegean Islands. Vol. 6. Edinburgh University Press, Edinburgh, 461-603.

Huber-Morath A (1981) Verbascum L. In: Rechinger KH (Ed.) Flora Iranica. Vol. 147. Akademische Drucku-Verlagsanstalt, Graz, 1-51.

IUCN (2001) IUCN Red List Categories and Criteria: Version 3.1: IUCN Species Survival Commission. IUCN, Gland, Switzerland \& Cambridge, UK.

Karavelioğulları FA, Duran A, Hamzaoğlu E (2004) Verbascum tuna-ekimii (Scrophulariaceae) a new species from Turkey. Annales Botanici Fennici 41: 227-231. 
Karavelioğulları FA, Vural M, Polat H (2006) Two new taxa from Central Anatolia Turkey. Israel Journal of Plant Sciences 54(2): 105-111. doi: 10.1560/IJPS_54_2_105

Karavelioğulları FA, Uzunhisarcıklı ME, Çelik S (2008a) Verbascum ozturkii (Scrophulariaceae), a new species from East Anatolia,Turkey. Pakistan Journal of Botany 40(4): 1595-1599.

Karavelioğulları FA, Aytaç Z (2008b) Revision of the genus Verbascum L. (Group A) in Turkey. Botany Research Journal 1(1): 9-32.

Karavelioğulları FA, Ocak A, Ekici M, Cabi E (2009a) Verbascum eskisehirensis sp. nov. (Scrophulariaceae) from central Anatolia,Turkey. Nordic Journal of Botany 27: 222-227.

Karavelioğulları FA (2009b) A new record Verbascum szovitsianum Boiss. var. szovitsianum (Scrophulariaceae) from Turkey. Biodicon 2(2): 68-70. doi: 10.1111/j.17561051.2008.00250.x

Karavelioğulları FA, Çelik S, Baser B, Yavru A (2011) Verbascum ergin-hamzolui (Scrophularıaceae), A New Species From South Anatolia, Turkey. Turkish Journal of Botany 35: 275-283.

Karavelioğulları FA (2012) Verbascum L. In: Güner A, Aslan S, Ekim T, Vural M, Babaç MT (Eds) Türkiye Bitkileri Listesi (Damarlı Bitkiler). Nezahat Gökyiğit Botanik Bahçesi ve Flora Araştırmaları Derneği Yayını, İstanbul, 850-870.

Karavelioğulları FA, Yüce E, Başer B (2012) Verbascum duzgunbabadagensis (Scrophulariaceae), a new species from eastern Anatolia, Turkey. Phytotaxa 181(1): 47-53. doi: 10.11646/ phytotaxa.181.1.3

Kaynak G, Daşkın R, Yılmaz O, Erdoğan E (2006) Verbascum yurtkurianum Scrophulariaceae) a new species from north west Anatolia, Turkey. Annales Botanici Fennici 43: 456-459.

Meikle RD (1985) Verbascum L. In: Meikle RD (Ed.) Flora of Cyprus. Vol. 2. Royal Botanic Gardens, Kew, 1196-1200.

Murberck S (1925) Monographie Der Gattung Celsia. Acta Universitatis Lundensis ser. 22(1): $1-239$.

Murberck S (1933) Monographie Der Gattung Verbascum. Acta Universitatis Lundensis ser. 29(2): 1-630.

Özhatay N (2006) Check-list of additional taxa to the Supplement Flora of Turkey III. Turkish Journal of Botany 30: 281-316.

Sutory K (2001) Two new hybrids of Verbascum from Turkey and Spain. Bocconea 13: 457-460.

Sutory K (2004) New hybrids of Verbascum (Scrophulariaceae) from Turkey. Turkish Journal of Botany 28: 261-262.

Vural M, Aydoğdu M (1993) A new species from central Anatolia Verbascum gypsicola (Scrophulariaceae). The Karaca Arboretum Magazine 2(2): 75-78.

Yilmaz G, Dane F (2008) Verbascum samniticum Ten. (Scrophulariaceae): A new record for the flora of Turkey. Turkish Journal of Botany 32(5): 411. 